\title{
Colesterol y triglicéridos sanguíneos en adolescentes durante el desarrollo sexual
}

\author{
Peter McColl C. ${ }^{1,2}$; Mariana Amador ${ }^{2}{ }^{2}$; Mirtha Díaz V. ${ }^{3}$ \\ Serum cholesterol and triglyceride in adolescents
}

\begin{abstract}
Cholesterol and triglyceride Serum concen trations were studied in 152 normal adolescents 153 males and 99 females\} at an adolescent's health center at Valparaiso. Chile. Tota: cholestero' serum fevels ranged from $120 \mathrm{mg} / \mathrm{dl} / 10 \mathrm{th}$ percentile) throughout $220 \mathrm{mg} \cdot \mathrm{dl}$ (90th percentile) and $20.4 \%$ of values for biood cholesterol were above 200 $\mathrm{mg} / \mathrm{dl}$. Triglyceride serum concentrations ranged from $57 \mathrm{mg} / \mathrm{dl}$ (10th percentile) throughout $161 \mathrm{mg} / \mathrm{dl}$ (90th percentidel and $11.9 \%$ of samples triglyceride levels above $150 \mathrm{mg} / \mathrm{dl}$. There was only a slight tendency of associa tion between breast stages 4 and 5 according to Tanner and high cholesterol serum leveis. There were positive relationships between cholesterol serum levels and body mass index $(p<0.001)$ and also with family history of coronary heart disease $\{p<0.02$.

(Key words: cholesterol, coronary heart disease, adolescents\}).
\end{abstract}

Las enfermedades cardiovasculares constitu. yen è Chile, como en paises desarrollados, la primera causa de muerte ( $30 \%$ del total) ${ }^{1}$.

Diversos estudios han demostrado que en la génesis de las enfermedades cardiovasculares participan tanto factores que pueden ser modi. ficados como otros que no pueden serjo. Entre los últimos figuran la herencia, el sexo, la raza y la edad. Entre los modificables se encuentran la presión arterial alta, las concentraciones elevadas de lípidos sanguineos, la diabetes mellitus, el tabaquismo y la dieta $a^{2,3}$.

Puesto que la mayor parte de los factores de riesgo descritos tiene algún grado de expresión en la adolescencia, el conocimiento de su existencia y de sus relaciones con otros hechos del desarrollo, la nutrición y la historia familiar pueden tener gran importancia en el diseño y aplicación de estrategias de control y prevención opartunos.

Entre los criterios de riesgo más empleados están las concentraciones séricas de colesterol y triglicéridos, ciertos parámetros nutricionales, como el índice de masa corporal (IMC) de Quetelet y los antecedentes familiares de enfermedad coronaria e hiperlipidemia.

1. Consultorio Integral del Adolescente, Valparaíso.

2. Instituto Médico Laennec, Viña del Mar.

3. Analista de sistemas de la Unidad de Salud Familiar, INTA, Universidad de Chile.
En efecto, el IMC se correlaciona positivamente con las concentraciones de colesterol y triglicérjdos en el suero ${ }^{2}$, cuya correcta interpretación, en la adolescencia, hace necesaria la debida consideración a la etapa del desarrollo puberal correspondiente ${ }^{4,5}$, para lo cual son útiles los criterios de la clasificación de Tanner. La inclusión de antecedentes parentales de enfermedad coronaria entre estos factores, obedece al alto riesgo de ocurrencia de la misma en el futuro, asi como de anomalias de los lípidos y las lipoproteinas plasmáticas en niños con historia familiar de dicha afección ${ }^{6,7}$.

El propósito del estudio, cuyos resultados se presentan a continuación, fue describir la distribución de las concentraciones séricas de colesterol $y$ triglicéridos en adolescentes chilenos sanos y la forma en que ellas se relacionan con las etapas de desarollo puberal, el índice de masa corporal y los antecedentes familiares de enfermedad coronaria.

\section{Material y Método}

Se estudiaron 152 adolescentes sanos ( 53 varones y 99 mujeres) entre 13 y 19 años de edad, beneficiarios del Sistema Nacional de Servicios de Salud, que acudieron para control de salud al Consultorio lntegral del Adolescente, Valparaíso. Todos ellos tenían apellidos de origen espanol.

Se evaluó el estado nutricional mediante peso en balanza de palanca con intervalos de $100 \mathrm{~g}$, talla con pedómetro metálico vertical, perimetro braquial con 
cinta métrica flexible, pliegues cutáneos con calibrador Lange. El peso y la talla se utilizaron para calcular el indice de masa corporal de Quetelet, dividiendo el peso actual por el cuadrado de la talla. Dicho índice guarda muy buena relación con la cantidad de grasa corporal cajculada por densitometría, ?. El grado de desarrollo puberal se evaluó según tablas de Tanner. Se midieron los niveles de colesteroi total y triglicéridos con método enzimático de Boehringer-Mannheim. Se efectuó una encuesta dirigida acerca de antecedentes familiares de enfermedades cardiovasculares, tabaquismo, diabetes mellitus, hipertensión arteriai $y$ obes1dad.

El procesamiento de la información se hizo en la terminal INTA2 del computador IBM370-43B1 deI Servicio de Computación e Informática de la Universidad de Chile (SECI), utilizando la prueba de Chi cuadrado. Las categorías de dispersión de las concentraciones séricas de colesterol y triglicéridos seleccionadas se confeccionaron de acuerdo al punto de mayor fuerza de asociación de las variables. Estos puntos coinciden con los valores que propone el Comité de Nutrición de la Academia Americana de Pediatría ${ }^{20}$.

\section{Resultados}

Las concentraciones séricas de colesterol total fluctuaron entre $120 \mathrm{mg} / \mathrm{dl}$ (percentil 10) y $261 \mathrm{mg} / \mathrm{dl}$ (percentil 80) (tabla 1). Las de trigli-

Tabla 1

Concentraciones de colesterol total en el suero en 99 mujeres $y 53$ vatones adolescentes

\begin{tabular}{ccc}
\hline $\begin{array}{c}\text { Colesterol total } \\
\text { mg/dl }\end{array}$ & $\mathrm{n}^{\text {Adolescentes }}$ & $\%$ \\
\hline \multirow{6}{*}{176} & 97 & 63,8 \\
$177-199$ & 24 & 15,8 \\
$\geqslant 200$ & 31 & 20,4 \\
Total & 152 & 100,0 \\
\hline
\end{tabular}

céridos fluctuaron entre $57 \mathrm{mg} /$ dl (percentil 10) y $161 \mathrm{mg} / \mathrm{dl}$ (percentil 90 ); $11,9 \%$ de las muestras terian concentraciones sobre 150 $\mathrm{mg} / \mathrm{d}$ (percentil 88) (tabla 2).

La distribución de las concentraciones séricas de colesterol y triglicéridos entre varones y mujeres no mostro diferencias significativas.

En el sexo femenino se estudiaron el grado de desarrollo mamario y la distribución del vello pubiano según criterios de Tanner y se investigó su posible asociación con los niveles de lípidos sanguineos. Sólo se observó una tendencia a la asociación entre las concentraciones séricas de colesterol total y el grado de desarrollo mamario (tabla 3). En los varones el tamaño genital y el grado de distribución del vello pubiano no mos. traron asociación con las concentracjones séricas de lípidos.

Con respecto al estado nutricional se ubservó una asociación altamente significativa entre concentraciones de colesterol total e indice de masa corporal $(\mathrm{p}<0,01)$ (tabla 4 ), pero no se encontró asociación entre área grasa braquial y dichas concentraciones séricas.

De los anteredentes mórbidos familiares investigados, la enfermedad coronaria presentó

Tabla 3

Tabla 2

Concentraciones séricas de triglicéridos en 151 adolescentes de ambos sexos

\begin{tabular}{|c|c|c|}
\hline \multirow{2}{*}{$\begin{array}{c}\text { Triglicéridos } \\
\text { mg/dl }\end{array}$} & \multicolumn{2}{|c|}{ Adolescentes } \\
\hline & $\mathbf{n}$ & $\%$ \\
\hline $\begin{array}{l}\leqslant 150 \\
\geqslant 151\end{array}$ & $\begin{array}{r}133 \\
18\end{array}$ & $\begin{array}{l}88,1 \\
11,9\end{array}$ \\
\hline Total & 151 & 100,0 \\
\hline
\end{tabular}

Sin información $=1$ caso.

Tabla 3

Concentraciones séricas de colesterol y grado de desarrollo mamatio según Tanner en 99 mujeres adolescentes

\begin{tabular}{|c|c|c|c|c|}
\hline \multirow[t]{2}{*}{$\begin{array}{c}\text { Grado de } \\
\text { desarrollo mamario }\end{array}$} & \multicolumn{2}{|c|}{$\begin{array}{c}\text { Niveles de colesterol } \\
\qquad 176\end{array}$} & \multicolumn{2}{|c|}{$\begin{array}{c}\text { Total mg · dl } \\
177\end{array}$} \\
\hline & n & $\%$ & $n$ & $\%$ \\
\hline $\begin{array}{l}1-2-3 \\
4-5\end{array}$ & $\begin{array}{l}12 \\
49\end{array}$ & $\begin{array}{l}19,7 \\
80,3\end{array}$ & $\begin{array}{l}13 \\
24\end{array}$ & $\begin{array}{l}35,1 \\
64,9\end{array}$ \\
\hline Total & 61 & 100,0 & 37 & 100,0 \\
\hline
\end{tabular}


Tabla 4

Concentraciones séricas de colesterol total según índice de masa corporal en $\mathbf{1 5 2}$ adolescentes de ambos sexos

\begin{tabular}{|c|c|c|c|c|}
\hline \multirow{3}{*}{$\begin{array}{l}\text { Colesterol total } \\
\mathrm{mg} / \mathrm{dl}\end{array}$} & \multicolumn{4}{|c|}{ Indice masa corporal $\left(\mathrm{kg} / \mathrm{m}^{2}\right)$} \\
\hline & \multicolumn{2}{|c|}{$<25$} & \multicolumn{2}{|c|}{$>25$} \\
\hline & $\mathbf{n}$ & $\%$ & $\mathrm{n}$ & $\%$ \\
\hline $\begin{array}{l}\leqslant 176 \\
\geqslant 177\end{array}$ & $\begin{array}{l}79 \\
36\end{array}$ & $\begin{array}{l}68,7 \\
31,3\end{array}$ & $\begin{array}{l}17 \\
19\end{array}$ & $\begin{array}{l}47,2 \\
52,8\end{array}$ \\
\hline Total & 115 & 100,0 & 36 & 100,0 \\
\hline
\end{tabular}

$\mathrm{p}<0,01$

asuciación estadística con los tiveles de colesterol elevados (tabla 5). No se encontró asociación estadística de niveles de colesterol y triglicéridos con antecedente familiar de obesidad. hipertensión arterial, diabetes mellitus y tabaquismo.

\section{Discusión}

En trabajos previos se han descrito los cambios que sufren las concentraciones de lípidos sanguineos durante la pubertad ${ }^{4}, 5$. En los adolescentes aquí descritos sólo encontramos una tendencia a la asociación de concentraciones mayores de colesterol con el grado de desarro. lo mamario, sin detectar diferencias entre los sexos, to que hace suponer que las actividades hormonales no tendrían una relación definida con las concentraciones de colesterol y triglicéridos. No existe un signo de desarrollo pube. ral que sea útil como indicador de niveles de lipidos. Los antecedentes familiares de enfermedad coronaria son muy importantes, pues se encontró asociación significativa de ellos con con- centraciones altas de colesterol. Tal asociación podria deberse a factores genéticos, ya que no se encontró relación entre las concentraciones elevadas de colesterol y triglicéridos en los adolescentes y antecedentes familiares de obesi. dad. Sin embargo, no es posible descartar la influencia de los hábitos alimentarios de la familia, pues se han descrito que las concentraciones de lipoproteínas plasmáticas varian de acuerdo a la dieta en distintos paises ${ }^{11}$. Para establecer una relación entre los hábitos alimentarios y los niveles de colesterol y triglicéridos serían nece. sarias encuestas de alimentación que identifiquen el tipo y la cantidad de ácidos grasos consumidos, que en este caso no se realizaron.

En adultos jóvenes se ha encontrado una relación positiva entre el IMC y las concentraciones de colesterol $y$ triglicéridos ${ }^{2}$. En nuestra sarie se encontró tal relación sólo para colesterol.

El IMC es un indicador nutricional fácil de calcular, pues sólo se necesita conocer el peso y la talla del paciente. Cuando el valor de este indice en un adolescente es mayor de $25 \mathrm{~kg} / \mathrm{m}^{2}$

Tabla 5

Concentraciones de colestetol total en el suero según antecedente familiar de enfermedad cotonaria en 152 adolescentes

\begin{tabular}{|c|c|c|c|c|}
\hline \multirow{3}{*}{$\begin{array}{c}\text { Colesterol total } \\
\mathrm{mg} / \mathrm{dl}\end{array}$} & \multicolumn{4}{|c|}{ Antecedente familiar de enfermedad coronaria } \\
\hline & & & & \\
\hline & $\mathrm{n}$ & $\%$ & n & $\%$ \\
\hline$\leqslant 176$ & 86 & 67,7 & 11 & 44 \\
\hline$\geqslant 177$ & 41 & 32,3 & 14 & 56 \\
\hline Total & 127 & 110,0 & 25 & 100,0 \\
\hline
\end{tabular}

$\mathrm{p}<0,02$. 
o existe el antecedente familiar de enfermedad coronaria, se debe considerar que el adolescente tiene alto riesgo de concentraciones elevadas de colesterol y triglicéridos elevados y estudiarle los lípidos sanguineos.

\section{Resumen}

Se midieron las concentraciones séricas de colesterol y triglicéridios en 152 adolescentes ( 53 varones y 99 mujeres) que acudieron a control de salud al Consultorio Integral de Adolescentes Valparaíso. Las concentraciones de colesterol total fluctuaron entre $120 \mathrm{mg} / \mathrm{dl}$ (percentil 10) y $220 \mathrm{mg} / \mathrm{dl}$ (percentil 90 ) y $20,4 \%$ estaban sobre $200 \mathrm{mg} / \mathrm{dl}$. Las de triglicéridos fluctuaron entre $57 \mathrm{mg} / \mathrm{dl}$ (percentil 10) y $161 \mathrm{mg} / \mathrm{dl}$ (percentil 90) siendo $11,9 \%$ mayores que $150 \mathrm{mg} / \mathrm{dl}$. Sólo se encontró una tendencia a la asociación entre concentraciones de colesterol total y desarrollo mamario, en cambio se encontró asocia. ción clara entre colesterol e índice de masa cor. poral $(\rho<0,01)$ y antecedente familiar de enfermedad coronaria $(\mathrm{p}<0,02)$.

(Palabras clave: colesterol sérico, triglicéridos séricos, masa corporal, enfermedad coronaria familiar.)

\section{Agradecimientos}

A las enfermeras señoras Vinka Montero y Raquel Aguirre, por su colaboración en la realización de este estudio.

\section{Referencias}

1. Ministerio de Salud: Psoyecciones de Morbilidad y Mortalidad de Chile al mediano plazo. 1983.

2. Danahue, R.: Orchard, T.; Kuller, L. y Drasch, A.: Lípidos y Lipoproteínas en la población adulta joven: en el Condado de Deaver. Am J Epidemiol $1985 ; 122: 458-467$.

3. Armesen, E. l' Forsdahl, A.: El estudio Cardiológico de Tromso: Factores de riesgo cotonario y su asociación con las condiciones de vida durante 1a infancia. J Epidemiol Community Health 1985; 39: 210-214.

4. Widhalm, K., Hozl, M. y Brubacher, G.: Lipids, Lipoproteins an Alpha-Tocopherol: Relationship and changes during Adolescence. Ann Nutr Metab $1985 ; 29: 12+18$.

5. Tell, G.; Mistelmark. M.: Vellar, O.: Cholesterol, High Density Lipoprotein cholesterol and triglicerides during puberty the Oslo. Young study. Am J Epiqemiol $1985 ; 122: 750-761$.

6. Lee, I..; Lawer, $R$. ' Clark, W.: Lipoproteínas en la patogenia de hombres con enfermedad arterial coronaria: niños con mayor riesgo. Pediatrics $1986 ; 78: 330-337$.

7. Levine, R.; Hennekens, C. Rosner, B. el al.: Factores de rjesgo cardiovascular en hijos de hombres con infarto de míocardio temprano. Pub Health Rep 1981; 96: 58-60.

8. Hontwast, J.G. y Deurenberg, P.: The risk of obesity, Epidemiological studies (abstract). En: First International Meeting on Body Weight control Monteaux, $1-4$ abrik, 1985.

9. Brunzell, $J_{\text {.: }}$ Are all obese patients at risk for cardiovascular disease? Int I Obesity 1984; 8: $571-578$

10. Indications for cholesterol Testing in children. Commirtee on Nurrition. Pediatrics 1989; 83: 141-142.

11. Seven Countries Study: Atherosclerosis Reviews. Vol, 7. Raven Press. New York, 1980; 7; 120-126. 